\title{
Puerarin Suppress Apoptosis of Human Osteoblasts via ERK Signaling Pathway
}

\author{
Ling-juan Liu, ${ }^{1}$ Li-qun Liu, ${ }^{1}$ Tao Bo, ${ }^{1}$ Shi-jun Li, ${ }^{1}$ Zhen Zhu, \\ Rong-rong Cui, ${ }^{2}$ and Ding-an Mao ${ }^{1}$ \\ ${ }^{1}$ Department of Pediatrics, The Second Xiang-Ya Hospital, Central South University, 139 Middle Renmin Road, \\ Changsha, Hunan 410011, China \\ ${ }^{2}$ Institute of Metabolism and Endocrinology, The Second Xiang-Ya Hospital, Central South University, \\ 139 Middle Renmin Road, Changsha, Hunan 410011, China
}

Correspondence should be addressed to Rong-rong Cui; rongrongcui530@hotmail.com and Ding-an Mao; mda5611@sina.com Received 3 January 2013; Revised 31 March 2013; Accepted 10 April 2013

Academic Editor: Guang-Da Xiang

Copyright (C) 2013 Ling-juan Liu et al. This is an open access article distributed under the Creative Commons Attribution License, which permits unrestricted use, distribution, and reproduction in any medium, provided the original work is properly cited.

\begin{abstract}
Puerarin, the main isoflavone glycoside extracted from Radix Puerariae, is an isoflavone traditional Chinese herb. Previous studies have demonstrated that puerarin could regulate osteoblast proliferation and differentiation to promote bone formation. However, the effect of puerarin on the process of human osteoblasts (hOBs) apoptosis is still unclear. In this study, we detected the function of puerarin on serum-free-induced cell apoptosis using ELISA and TUNEL arrays and then found that the mortality of hOBs was significantly decreased after exposure to $10^{-10}-10^{-6} \mathrm{M}$ puerarin and reached the maximal antiapoptotic effect at the concentration of $10^{-8} \mathrm{M}$. In addition, compared with the control group, puerarin notably increased the Bcl-2 protein levels while it decreased the Bax protein levels in the hOBs in a dose-dependent way. $10^{-7} \mathrm{M}$ puerarin decreased the Bax/Bcl-2 ratio with a maximal decrease to 0.08. Moreover, puerarin activated ERK signaling pathways in hOBs, and the antiapoptotic effect induced by puerarin was abolished by incubation of ERK inhibitor PD98059. Similarly, the estrogen receptor antagonist ICI182780 also suppressed the inhibitory effect of puerarin on hOBs apoptosis. In conclusion, puerarin could prevent hOBs apoptosis via ERK signaling pathway, which might be effective in providing protection against bone loss and bone remolding associated with osteoporosis.
\end{abstract}

\section{Introduction}

Osteoblasts, mononuclear specialized cells derived from mesenchymal precursor cells, are responsible for bone formation, deposition, and mineralization $[1,2]$, playing an essential role in the maintenance of the stability of bone microarchitecture. Osteoblast apoptosis, induced by various pathological and physiological factors (e.g., estrogen loss, glucocorticoids, weightlessness, and aging), breeds a series of bone disorders. Osteoporosis is the most prevalent bone disorder affecting the elders, which is characterized by an imbalance between bone formation and bone resorption [3-5]. Several studies have showed that apoptosis might be the third most common cause of osteoporosis, and $60-80 \%$ of osteoblasts were estimated to originally assembled at the resorption pit die by apoptosis [6]. Therefore, apoptosis is generally served as the pivotal target for prevention and/or ameliorate osteoporosis.
Puerarin, 7-hydroxy-3-(4-hydroxyphenyl)-1-benzopyran-4-one 8 - $\beta$-D-glucopyranoside $\left(\mathrm{C}_{12} \mathrm{H}_{20} \mathrm{C}_{9}\right)$, is one of the major isoflavonoid compounds extracted from the root of a wild leguminous creeper $[7,8]$. It possesses estrogen-like structure and moderates estrogenic activity. As a famous phytoestrogen, current studies have established that puerarin provides a strong protection against osteoporosis through facilitating osteoblast proliferation and differentiation $[9,10]$. Although it acts as an important regulatory factor for cell death, the role of puerarin on osteoblast apoptosis and its underlying mechanism of action are still unclear. As we know osteoblasts apoptosis is an extremely complicated event; a series of proteins and signaling pathways (e.g., Bcl-2 family proteins, ERK, MAPK, APJ/PI3-K/Akt, JAK2, and Fas) are reported to be involved in this process in vitro and in vivo [11-15]. In present study, we aim to detect the impact of puerarin on the serum-deprivation-induced hOBs apoptosis 
along with observing the expression of $\mathrm{Bcl}-2$ and Bax protein using western blotting and the cell signal pathway involved.

\section{Materials and Methods}

2.1. Reagents. Puerarin and $17 \beta$-estradiol were purchased from Sigma, Inc. (USA). $\beta$-actin, anti-Bcl-2, Bax, ERK, pERK antibodies, anti-mouse, and -rabbit IgG peroxidase conjugate antibodies were purchased from Santa Cruz Biotechnology Inc. (Waltham, MA, USA). ICI 182780 and PD98059 were purchased from Calbiochem Corp. (San Diego, CA, USA).

2.2. Cell Cultures. hOBs were obtained from traffic accident victims suffering surgery, and this procedure was approved by the local research ethics committee as previously mentioned [16]. Cells isolated from femur were cultured in aMEM medium (Gibico-BRL. Corp., NY, USA) containing $15 \%$ fetal bovine serum, $100 \mathrm{U} / \mathrm{mL}$ penicillin, and $100 \mu \mathrm{g} / \mathrm{mL}$ streptomycin at $37^{\circ} \mathrm{C}$ in a $5 \% \mathrm{CO}_{2}$ atmosphere. Cells were identified as osteoblasts by the expression of ALP, collagen type I and osteocalcin, and formation of mineralized nodules as lately described [12].

To determine the effect of puerarin on apoptosis of hOBs, the cells were seeded for $24 \mathrm{~h}$ followed by culturing for $24 \mathrm{~h}$ in serum-free medium, then treated with $0,10^{-9} \mathrm{M}$, $10^{-8} \mathrm{M}, 10^{-7} \mathrm{M}$ puerarin, or $17 \beta$-estradiol $(\mathrm{E} 2,10 \mu \mathrm{M})$ for $48 \mathrm{~h}$. To analyze the effect of ERK or estrogen receptor (ER) inhibition on hOB apoptosis, hOBs were pretreated with $10 \mu \mathrm{M}$ ERK inhibitor PD98059 or ER antagonist ICI 182780 for $3 \mathrm{~h}$ anterior to incubation with $10^{-8} \mathrm{M}$ puerarin for $48 \mathrm{~h}$.

2.3. Western Blot Analysis. Immunoblotting was carried out as before [17]. Total protein was extracted with RIPA lysis buffer (Beyotime, China), then protein concentration determined by a Bradford assay, and equal amounts of protein were loaded onto SDS-PAGE and then transferred to PVDF membranes (Invitrogen, Carlsbad, CA, USA). Thereafter, the membranes were blocked with 5\% nonfat milk in PBS for $1 \mathrm{~h}$ at room temperature and then incubated with antiBcl-2, anti-Bax $(1: 500)$, or anti- $\beta$-actin $(1: 1000)$ antibodies overnight at $4^{\circ} \mathrm{C}$. Resultant protein bands after incubation with a proper secondary antibody were visualized by chemiluminescence. The absorbance values of target proteins were analyzed through Gel-Pro 4.0 gel image analysis software. The absorbance ratio of each protein to internal reference was represented as the relative amount of target proteins [18].

2.4. Measurement of ERK Activation. hOBs were treated with $10^{-8} \mathrm{M}$ puerarin for $0-45 \mathrm{~min}$. The cell layers were washed twice with cold PBS and then lysed with a buffer consisting of $20 \mathrm{mM}$ Tris- $\mathrm{HCl}$ ( $\mathrm{pH} 7.5$ ), $150 \mathrm{mM} \mathrm{NaCl}, 1 \%$ Triton X-100, $10 \mathrm{mM} \mathrm{NaH}_{2} \mathrm{PO}_{4}, 10 \%$ glycerol, $2 \mathrm{mM} \mathrm{Na}_{3} \mathrm{VO}_{4}, 10 \mathrm{mM} \mathrm{NaF}$, $1 \mathrm{mM}$ ABSF, $10 \mu \mathrm{g} / \mathrm{mL}$ leupeptin, and $10 \mu \mathrm{g} / \mathrm{mL}$ aprotinin. Western blot analysis was carried out as previously described $[19,20]$. Equal amount of proteins were transferred onto
PVDF membranes, then incubated with anti-ERK or anti-pERK monoclonal antibodies (1:500). The ECL detection kit was used for detection.

\subsection{Measurement of Cell Apoptosis}

2.5.1. Cell Death ELISA Detection. Cell death ELISA detection was performed for detecting the apoptosis levels as previously described [21]. Cell death detection ELISA kit (Roche Diagnostics GmbH, Roche Molecular Biochemicals, Mannheim, Germany) was used for detection, according to the kit protocol. Briefly, cells were plated at a density of 10,000 cells/well in 24-well plates for 1 day followed by culture in serum-free medium for $48 \mathrm{~h}$ in the absence or presence of $0-10^{-6} \mathrm{M}$ puerarin or $10 \mu \mathrm{M}$ E2. Cells were rinsed with PBS and incubated with $0.5 \mathrm{~mL}$ of lysis buffer at $4^{\circ} \mathrm{C}$ for $30 \mathrm{~min}$, then centrifuged for $10 \mathrm{~min}$ at $15,000 \mathrm{rpm}$. Aliquots of the supernatant were tested for the rate of apoptosis through the cell death detection kit.

2.5.2. TUNEL Assay. Terminal deoxynucleotidyl transferasemediated deoxyribonucleotide triphosphate nick endlabeling (TUNEL) was generally used to assess cell death. hOBs were washed with PBS after cultured in serumdeprivation medium for $48 \mathrm{~h}$ in the absence or presence of $10^{-8} \mathrm{M}$ puerarin and then fixed with $4 \%$ paraformaldehyde for $30 \mathrm{~min}$ at room temperature. Thereafter, the cells were incubated with the TUNEL reaction mixture(Roche Molecular Biochemicals, Indianapolis, IN) for $60 \mathrm{~min}$ at $37^{\circ} \mathrm{C}$ followed by labeling with fluorescein isothio cyanate (FITC)-conjugated anti-fluorescein anti-goat antibody (Fab fragment) for an additional $30 \mathrm{~min}$. The nuclei were counterstained with 4,6-diamidino-2-phenylindole (DAPI). Finally, TUNEL-positive cells were photographed on an Olympus microscope.

2.6. Statistical Analysis. The data are presented as mean \pm standard deviation (SD). Statistical analyses of the data were performed through one-way analysis of variance (ANOVA) and the LSD post hoc test for multiple comparisons. $P<$ 0.05 was considered the statistical significant difference. All experiments were repeated at least three times.

\section{Results}

3.1. Puerarin Protects the hOBs from Serum-Free-Induced Apoptosis. Our study used ELISA assays to assess hOBs apoptosis cultured in serum-depravation medium for $48 \mathrm{~h}$ in the presence of $0-10^{-6} \mathrm{M}$ puerarin or $10 \mu \mathrm{M}$ E2. Results showed that after exposure to puerarin the apoptotic cells at $10^{-10} \mathrm{M}\left(2.23 \pm 0.14\right.$ ELISA absorbance units), $10^{-9} \mathrm{M}(1.89 \pm$ 0.16 ELISA absorbance units), $10^{-8} \mathrm{M}(1.54 \pm 0.13$ ELISA absorbance units), $10^{-7} \mathrm{M}$ (1.62 \pm 0.15 ELISA absorbance units), and $10^{-6} \mathrm{M}(1.58 \pm 0.12$ ELISA absorbance units) puerarin were less than those of the negative control group $(2.51 \pm 0.11$ ELISA absorbance units, all $P<0.05)$, while they were higher than the positive group exposed to $10 \mu \mathrm{M}$ E2 $(1.30 \pm 0.12$ ELISA absorbance units, $P<0.05)$. Puerarin 


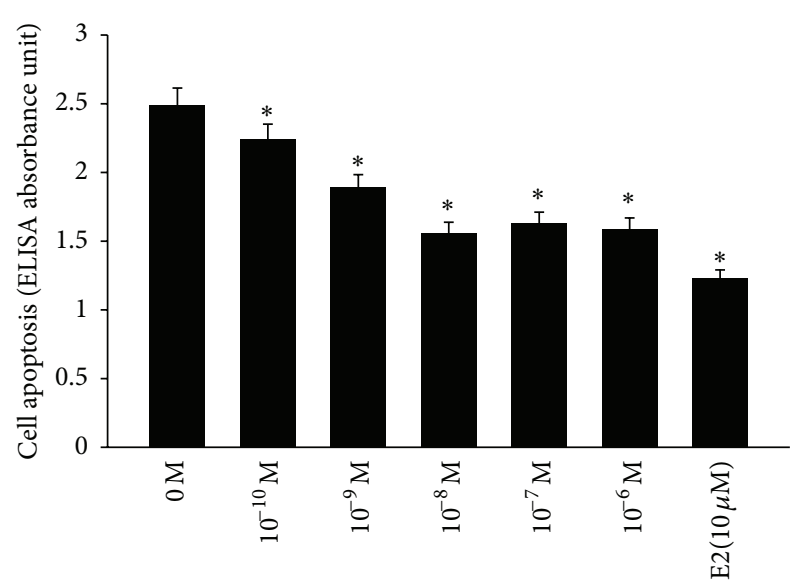

FIgure 1: Puerarin protected the hOBs from serum-free-induced apoptosis. Cells were treated with $0-10^{-6} \mathrm{M}$ puerarin, and cells exposed to $10 \mu \mathrm{M}$ E2 served as the positive control. Apoptosis were measured by ELISA according to the kit specification. The bars represent the mean $\pm \mathrm{SD}(n=6),{ }^{*} P<0.05$, as compared with the control cells.

reached the maximal antiapoptotic effect at the concentration of $10^{-8} \mathrm{M}$ (Figure 1). The results of TUNEL assay also indicated that $10^{-8} \mathrm{M}$ puerarin significantly decreases hOBs apoptosis caused by serum deprivation compared to the control $(P<0.05$, Figure 2$)$.

3.2. Effects of Puerarin on the Expression of Bcl-2 and Bax in hOBs. Bcl-2 and Bax are the essential members of Bcl-2 family which involved in the process of apoptosis. Western blot analysis was used to detect the expression of Bcl-2 and Bax in hOBs incubated with $0,10^{-9} \mathrm{M}, 10^{-8} \mathrm{M}$, and $10^{-7} \mathrm{M}$ puerarin. As a result, we found that puerarin increased the levels of Bcl-2 protein, while it decreased the expression of Bax in hOBs in a dose-dependent manner (all $P<0.05$, Figure 3 ). The $\mathrm{Bax} / \mathrm{Bcl}-2$ ratio was set to 1 in the control group, and $10^{-7} \mathrm{M}$ puerarin could downregulate the $\mathrm{Bax} / \mathrm{Bcl}-2$ ratio with a maximal decrease to 0.08 (all $P<0.05$, Figure 3 ).

\subsection{Puerarin Activated ERK Signaling Pathway in hOBs.} ERK signaling cascades is a classic pathway involved in the regulation of cell death. According to our results, $10^{-8} \mathrm{M}$ was the optimum concentration for puerarin to produce the best antiapoptotic protection. Therefore, we used western blotting to confirm the effects of $10^{-8} \mathrm{M}$ puerarin on ERK phosphorylation and found that the levels of phosphorylated ERK was upregulated after $5 \mathrm{~min}$ incubation with puerarin compared with the control group $(P<0.05)$. Additionally, this effects was time-dependent with the peak activation of ERK at $45 \mathrm{~min}$ of incubation $(P<0.05$, Figure 4$)$.

\subsection{ERK Signaling Pathway and Estrogen Receptors Mediated} the Antiapoptotic Effects of Puerarin in hOBs. After detecting that puerarin could activate ERK signaling pathway in hOBs, we further verified whether the puerarin-mediated activation of ERK participates in apoptosis. We examined apoptosis

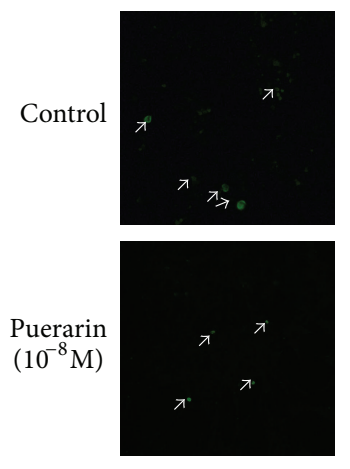

TUNEL
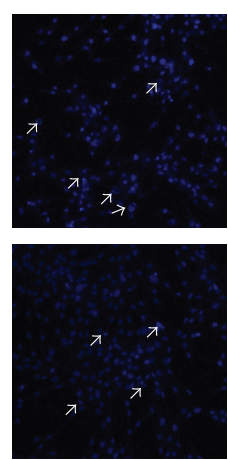

DAPI
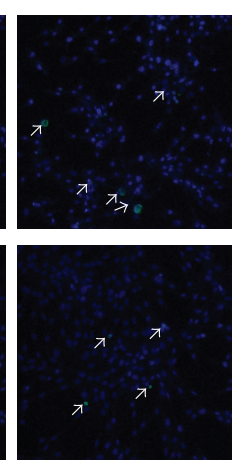

MERGE
FIGURE 2: Effect of puerarin on apoptosis of hOBs measured by TUNEL assay. Cells were exposed to $10^{-8} \mathrm{M}$ puerarin for $48 \mathrm{~h}$, while the control group was incubated with serum-free medium for $48 \mathrm{~h}$. Apoptotic nuclei were detected by TdT-mediated dUTP nick end-labeling (TUNEL). Arrows show apoptotic cells. Original magnification $\times 100$.

after incubation with $10^{-8} \mathrm{M}$ puerarin and/or PD98059 by ELISA, then detected that the protection effect of puerarin on hOBs was blocked by PD98059, which had little effects on apoptosis alone $(P<0.05$, Figure 5). Moreover, puerarin was proved to have estrogen-like structure and moderate estrogenic activity, and we found here that ICI 182780 did not affect the process of apoptosis but significantly suppressed the antiapoptotic effect of puerarin on hOBs $(P<0.05$, Figure 5$)$.

\section{Discussion}

Osteoblast apoptosis is generally regarded as a key component of bone turnover, repair, and regeneration [22]. It is reported that approximately $50-70 \%$ of osteoblasts undergo apoptosis during bone regeneration [23]. Puerarin, an isoflavone traditional Chinese herb, is reported to significantly facilitate the survival rate of osteoblasts, and the puerarin-treated rats also displayed a higher rate of bone formation in the osteoblast implants than the control, suggesting that puerarin might regulate osteoblast proliferation and differentiation to promote bone formation in osteoblast implants $[24,25]$. In present research, we demonstrated that puerarin alleviates apoptosis of hOBs induced by serum deprivation through activating ERK signaling pathway.

Cell apoptosis is an essential process in maintaining the homeostasis under normal conditions [26]. Members of the $\mathrm{Bcl}-2$ family, including $\mathrm{Bcl}-2$ and $\mathrm{Bax}$, are the main regulators of apoptosis which promote (Bax) or inhibit ( $\mathrm{Bcl}-2)$ apoptosis [27]. Each of them regulates apoptosis independently [28]. Furthermore, Bcl-2 protein forms heterodimer complexes with Bax proteins, leading to the release of cytochrome $\mathrm{C}$ from the mitochondria and induction of cell apoptosis [29]. Previous reporters have proved that puerarin acts on a variety of apoptosis through regulating the expression of Bcl-2 family proteins in vitro, including vascular endothelial cells, vascular smooth muscle cells, and human neurons [30-32]. Here we 


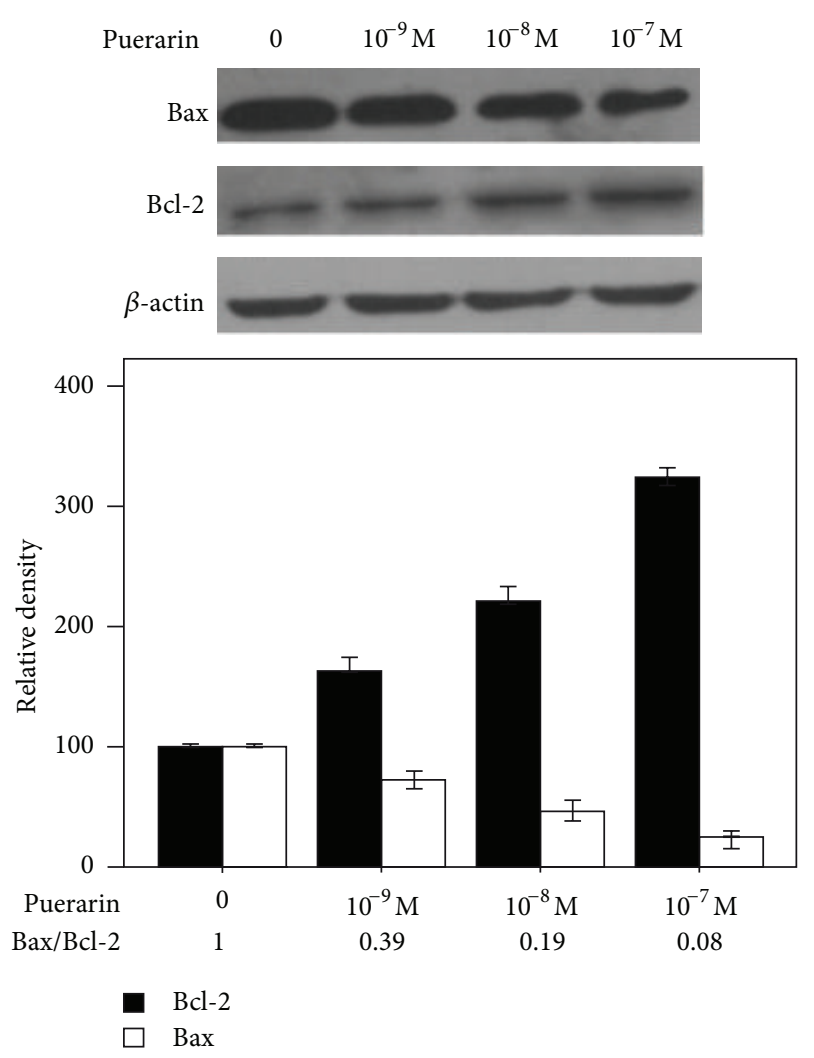

Figure 3: Effects of puerarin on the expression of Bcl-2 and Bax in hOBs. Cells were treated with $0,10^{-9} \mathrm{M}, 10^{-8} \mathrm{M}$, and $10^{-7} \mathrm{M}$ puerarin for $48 \mathrm{~h}$ before collecting proteins. Cell lysates were subjected to western blot analysis and incubated with anti-Bax, anti-Bcl-2, or anti- $\beta$-actin monoclonal antibodies. Gel-Pro 4.0 gel image analysis software was used to analyze the absorbance values of $\mathrm{Bcl}-2$ and $\mathrm{Bax}$.

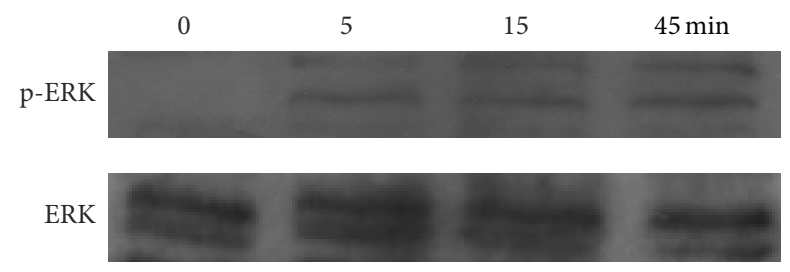

FIGURE 4: Effects of puerarin on ERK signaling pathways in hOBs. Anti-ERK and anti-p-ERK monoclonal antibodies were used to perform in western blotting. Cells were exposed to $10^{-8} \mathrm{M}$ puerarin for $0-45 \mathrm{~min}$. The levels of ERK and p-ERK were measured by densitometry of autoradiographs.

discovered that puerarin protects hOBs from serum-freeinduced apoptosis by upregulating the expression of $\mathrm{Bcl}-2$ and downregulating the expression of Bax and then significantly decreasing the $\mathrm{Bax} / \mathrm{Bcl}-2$ ratio in a dose-dependent manner, suggesting that the $\mathrm{Bcl}-2$ family participates in the regulation of the prevention hOBs from apoptosis by puerarin.

To gain further insight into the mechanisms by which puerarin suppresses hOBs apoptosis, we examined ERK signaling pathways. Extracellular signal-regulated kinase

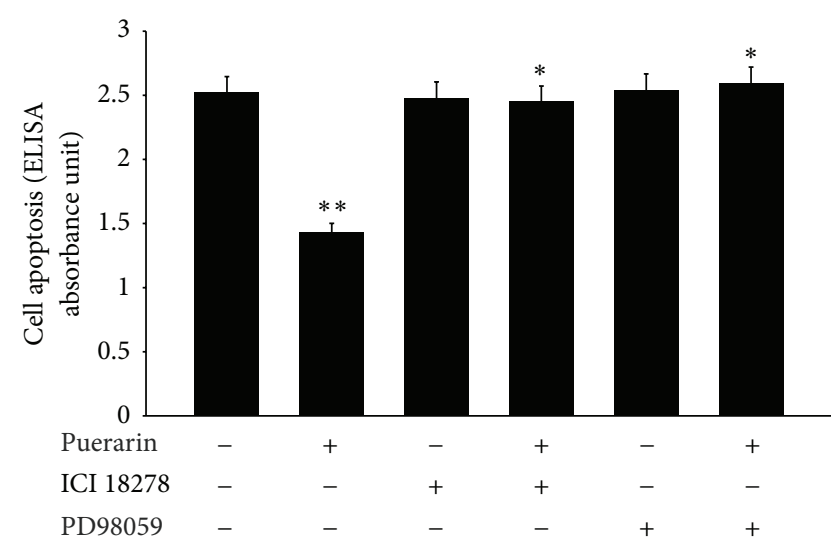

FIGURE 5: ERK signaling pathway and estrogen receptor mediated the antiapoptotic effects of puerarin in hOBs. Cells were incubated with PD98059 $(10 \mu \mathrm{M})$ and/or ICI $182780(10 \mu \mathrm{M})$ for $3 \mathrm{~h}$ prior to treatment with $10^{-8} \mathrm{M}$ puerarin for $48 \mathrm{~h}$. The bars represented the mean $\pm \mathrm{SD}(n=6) .{ }^{*} P<0.05$, as compared with the puerarintreated cells; ${ }^{* *} P<0.05$, as compared with the serum-free treated cells.

(ERK1/2), located at both the cytoplasm and the nucleus of cells, is a multifunctional serine/threonine kinases which induce various of substrates phosphorylation localized in all cellular compartments $[33,34]$. It is believed that ERK is involved mainly in the activation of nuclear transcription factors that control cell proliferation, differentiation and apoptosis [35]. Currently, osteoblasts apoptosis induced by serum deprivation was proved to be suppressed significantly through activating the ERK signaling pathway [12, 36]. Present study we investigated the effect of puerarin on ERK and found that puerarin activated ERK phosphorylation and inhibited hOBs apoptosis. This protection was eliminated through pretreatment with PD98059, indicating that ERK signaling pathway was the key link in the antiapoptotic effects of puerarin on hOBs.

Additionally, it is well known that ERs are the major targets for estrogen acting on osteoblasts apoptosis [37]. As a phytoestrogen, puerarin was reported to be closely connected with ERs. In the study of Tiyasatkulkovit et al. [38], ER antagonist ICI182780 substantially inhibited the facilitation of puerarin on osteoblast differentiation. These present findings corroborated that ICI182780 blocked the effects of puerarin on hOBs apoptosis just as was expected, supporting the conclusion that puerarin suppressed hOBs apoptosis in an ER-dependent manner.

\section{Conclusion}

The present study has confirmed the potential benefit of puerarin on hOBs apoptosis in vitro, which is mediated by ERK signaling pathway. It is well known that hOBs apoptosis plays an essential role in the process of osteoporosis. Consequently, we conclude here that puerarin might ameliorate bone loss and promote bone remolding after subjecting to osteoporosis. Further studies are needed to affirm the effect of puerarin on osteoporosis in vivo. 


\section{Acknowledgments}

This work was supported by Grants 81000313, 30900622, and 81070246 from the China National Natural Scientific Foundation; Grant 078202042 from Scholarship Award for Excellent Doctoral Student granted by Ministry of Education; Central South University "Excellent Doctoral Dissertation of training support program."

\section{References}

[1] H. Taipaleenmäki, L. Bjerre Hokland, L. Chen, S. Kauppinen, and M. Kassem, "Mechanisms in endocrinology: microRNAs: targets for enhancing osteoblast differentiation and bone formation," European Journal of Endocrinology, vol. 166, no. 3, pp. 359-371, 2012.

[2] C. V. Gurban and O. Mederle, "The OPG/RANKL system and zinc ions are promoters of bone remodeling by osteoblast proliferation in postmenopausal osteoporosis," Romanian Journal of Morphology and Embryology, vol. 52, supplement 3, pp. 11131119, 2011.

[3] N. Maruotti, A. Corrado, A. Neve, and F. P. Cantatore, "Bisphosphonates: effects on osteoblast," European Journal of Clinical Pharmacology, vol. 68, no. 7, pp. 1013-1018, 2012.

[4] A. Gohel, M. B. McCarthy, and G. Gronowicz, "Estrogen prevents glucocorticoid-induced apoptosis in osteoblasts in vivo and in vitro," Endocrinology, vol. 140, no. 11, pp. 5339-5347, 1999.

[5] H. Li, W. Qian, X. Weng et al., "Glucocorticoid receptor and sequential P53 activation by dexamethasone mediates apoptosis and cell cycle arrest of osteoblastic MC3T3-E1 cells," PLoS One, vol. 7, no. 6, Article ID e37030, 2012.

[6] S. C. Manolagas, "Birth and death of bone cells: basic regulatory mechanisms and implications for the pathogenesis and treatment of osteoporosis," Endocrine Reviews, vol. 21, no. 2, pp. 115137,2000

[7] F. S. Chueh, C. P. Chang, C. C. Chio, and M. T. Lin, "Puerarin acts through brain serotonergic mechanisms to induce thermal effects," Journal of Pharmacological Sciences, vol. 96, no. 4, pp. 420-427, 2004.

[8] R. M. Han, Y. X. Tian, E. M. Becker, M. L. Andersen, J. P. Zhang, and L. H. Skibsted, "Puerarin and conjugate bases as radical scavengers and antioxidants: molecular mechanism and synergism with $\beta$-carotene," Journal of Agricultural and Food Chemistry, vol. 55, no. 6, pp. 2384-2391, 2007.

[9] P. P. Wang, X. F. Zhu, L. Yang et al., "Puerarin stimulates osteoblasts differentiation and bone formation through estrogen receptor, p38 MAPK, and Wnt/ $\beta$-catenin pathways," Journal of Asian Natural Products Research, vol. 14, no. 9, pp. 897-905, 2012.

[10] M. Y. Zhang, H. Qiang, H. Q. Yang, X. Q. Dang, and K. Z. Wang, "In vitro and in vivo effects of puerarin on promotion of osteoblast bone formation," Chinese Journal of Integrative Medicine, vol. 18, no. 4, pp. 276-282, 2012.

[11] K. M. Wiren, A. R. Toombs, A. A. Semirale, and X. Zhang, "Osteoblast and osteocyte apoptosis associated with androgen action in bone: requirement of increased Bax/Bcl-2 ratio," Bone, vol. 38, no. 5, pp. 637-651, 2006.

[12] X. Zhu, Y. Jiang, P. F. Shan et al., "Vaspin attenuates the apoptosis of human osteoblasts through ERK signaling pathway," Amino Acids, vol. 44, no. 3, pp. 961-968, 2013.
[13] H. Xie, L. Q. Yuan, X. H. Luo et al., "Apelin suppresses apoptosis of human osteoblasts," Apoptosis, vol. 12, no. 1, pp. 247-254, 2007.

[14] O. Morales, M. K. R. Samuelsson, U. Lindgren, and L. A. Haldosén, "Effects of $1 \alpha, 25$-dihydroxyvitamin D3 and growth hormone on apoptosis and proliferation in UMR 106 osteoblastlike cells," Endocrinology, vol. 145, no. 1, pp. 87-94, 2004.

[15] G. Kogianni, V. Mann, F. Ebetino et al., "Fas/CD95 is associated with glucocorticoid-induced osteocyte apoptosis," Life Sciences, vol. 75, no. 24, pp. 2879-2895, 2004.

[16] Y. S. Liu, Y. Lu, W. Liu et al., "Connective tissue growth factor is a downstream mediator for preptin-induced proliferation and differentiation in human osteoblasts," Amino Acids, vol. 38, no. 3, pp. 763-769, 2010.

[17] L. Q. Yuan, W. Liu, R. R. Cui et al., “Taurine inhibits osteoclastogenesis through the taurine transporter," Amino Acids, vol. 39, no. 1, pp. 89-99, 2010.

[18] E. Bona, H. Hagberg, E. M. Løberg, R. Bågenholm, and M. Thoresen, "Protective effects of moderate hypothermia after neonatal hypoxia- ischemia: short- and long-term outcome," Pediatric Research, vol. 43, no. 6, pp. 738-745, 1998.

[19] P. F. Shan, Y. Lu, R. R. Cui, Y. Jiang, L. Q. Yuan, and E. Y. Liao, "Apelin attenuates the osteoblastic differentiation of vascular smooth muscle cells," PLoS One, vol. 6, no. 3, Article ID e17938, 2011.

[20] L. Q. Yuan, J. H. Zhu, H. W. Wang et al., "RANKL is a downstream mediator for insulin-induced osteoblastic differentiation of vascular smooth muscle cells," PLoS One, vol. 6, no. 12, Article ID e29037, 2011.

[21] R. R. Cui, D. A. Mao, L. Yi et al., "Apelin suppresses apoptosis of human vascular smooth muscle cells via APJ/PI3-K/Akt signaling pathways," Amino Acids, vol. 39, no. 5, pp. 1193-1200, 2010.

[22] J. M. Hock, V. Krishnan, J. E. Onyia et al., "Osteoblast apoptosis and bone turnover," Journal of Bone and Mineral Research, vol. 16, no. 6, pp. 975-984, 2001.

[23] G. M. Bran, J. Stern-Straeter, K. Hörmann, F. Riedel, and U. R. Goessler, "Apoptosis in bone for tissue engineering," Archives of Medical Research, vol. 39, no. 5, pp. 467-482, 2008.

[24] Y. P. Hwang and H. G. Jeong, "Mechanism of phytoestrogen puerarin-mediated cytoprotection following oxidative injury: estrogen receptor-dependent up-regulation of PI3K/Akt and HO-1," Toxicology and Applied Pharmacology, vol. 233, no. 3, pp. 371-381, 2008.

[25] M. Y. Zhang, H. Qiang, H. Q. Yang, X. Q. Dang, and K. Z. Wang, "In vitro and in vivo effects of puerarin on promotion of osteoblast bone formation," Chinese Journal of Integrative Medicine, vol. 18, no. 4, pp. 276-282, 2012.

[26] H. E. Abud, "Shaping developing tissues by apoptosis," Cell Death and Differentiation, vol. 11, no. 8, pp. 797-799, 2004.

[27] M. Bras, B. Queenan, and S. A. Susin, "Programmed cell death via mitochondria: different modes of dying," Biochemistry, vol. 70, no. 2, pp. 231-239, 2005.

[28] C. M. Knudson and S. J. Korsmeyer, "Bcl-2 and bax function independently to regulate cell death," Nature Genetics, vol. 16, no. 4, pp. 358-363, 1997.

[29] H. Zhang, Y. Liu, M. Lao, Z. Ma, and X. Yi, "Puerarin protects Alzheimer's disease neuronal cybrids from oxidant-stress induced apoptosis by inhibiting pro-death signaling pathways," Experimental Gerontology, vol. 46, no. 1, pp. 30-37, 2011. 
[30] R. L. Shi and J. J. Zhang, "Protective effect of puerarin on vascular endothelial cell apoptosis induced by chemical hypoxia in vitro," Yaoxue Xuebao, vol. 38, no. 2, pp. 103-107, 2003.

[31] L. H. Zhu, L. Wang, D. Wang et al., "Puerarin attenuates highglucose-and diabetes-induced vascular smooth muscle cell proliferation by blocking PKCbeta2/Rac1-dependent signaling," Free Radical Biology and Medicine, vol. 48, no. 4, pp. 471-482, 2010.

[32] J. Q. Han, K. Y. Yu, and M. He, "Effects of puerarin on the neurocyte apoptosis and p-Akt (Ser473) expressions in rats with cerebral ischemia/reperfusion injury," Zhongguo Zhong Xi Yi Jie He Za Zhi, vol. 32, no. 8, pp. 1069-1072, 2012.

[33] C. J. Tai, C. H. Lee, H. C. Chen et al., "High nuclear expression of phosphorylated extracellular signal-regulated kinase in tumor cells in colorectal glands is associated with poor outcome in colorectal cancer," Annals of Diagnostic Pathology, vol. 17, no. 2, pp. 165-171, 2013.

[34] M. Tasyriq, I. A. Najmuldeen, L. L. In et al., " $7 \alpha$-hydrox$\beta$-sitosterol from Chisocheton tomentosus induces apoptosis via dysregulation of cellular $\mathrm{Bax} / \mathrm{Bcl}-2$ ratio and cell cycle arrest by downregulating ERK1/2 activation," Evidence-Based Complementary and Alternative Medicine, vol. 2012, Article ID 765316, 12 pages, 2012.

[35] Q. H. Liang, Y. Jiang, X. Zhu et al., "Ghrelin attenuates the osteoblastic differentiation of vascular smooth muscle cells through the ERK pathway," PLoS One, vol. 7, no. 4, Article ID e33126, 2012.

[36] L. Y. Zhang, Y. Y. Zhou, F. Chen et al., "Taurine inhibits serum deprivation-induced osteoblast apoptosis via the taurine transporter/ERK signaling pathway," Brazilian Journal of Medical and Biological Research, vol. 44, no. 7, pp. 618-623, 2011.

[37] R. S. Weinstein, C. A. O’Brien, R. L. Jilka et al., "Estrogens attenuate oxidative stress and the differentiation and apoptosis of osteoblasts by DNA-binding-independent actions of the ER $\alpha$, Journal of Bone and Mineral Research, vol. 25, no. 4, pp. 769-781, 2010.

[38] W. Tiyasatkulkovit, N. Charoenphandhu, K. Wongdee et al., "Upregulation of osteoblastic differentiation marker mRNA expression in osteoblast-like UMR106 cells by puerarin and phytoestrogens from Pueraria mirifica," Phytomedicine, vol. 19, no. 13, pp. 1147-1155, 2012. 


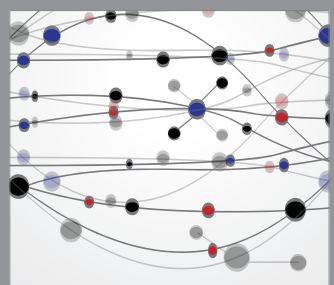

The Scientific World Journal
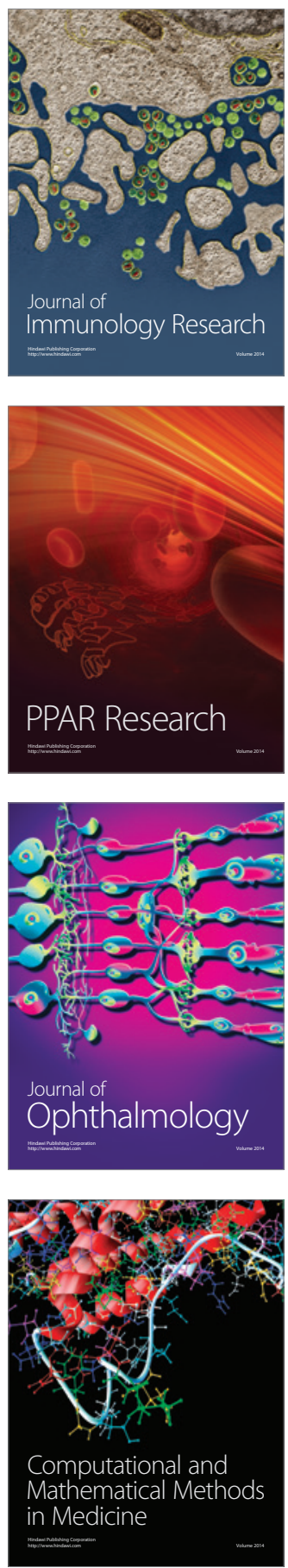

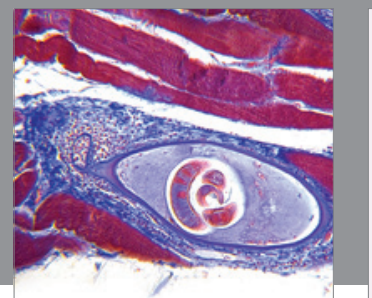

Gastroenterology

Research and Practice
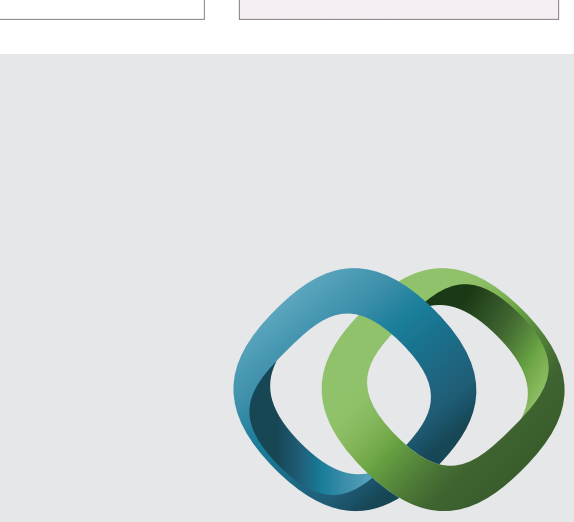

\section{Hindawi}

Submit your manuscripts at

http://www.hindawi.com
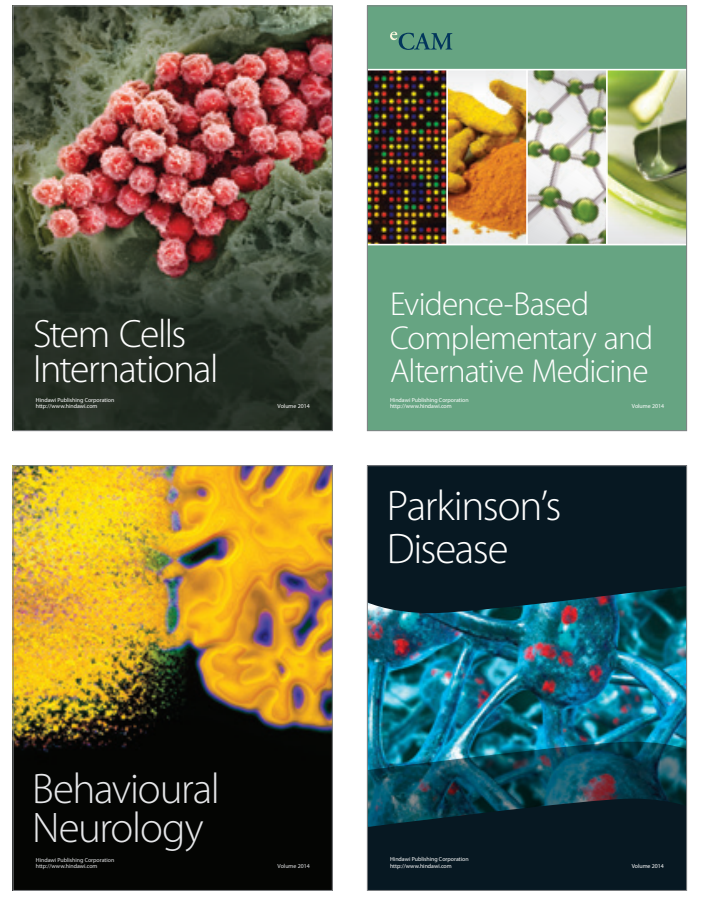
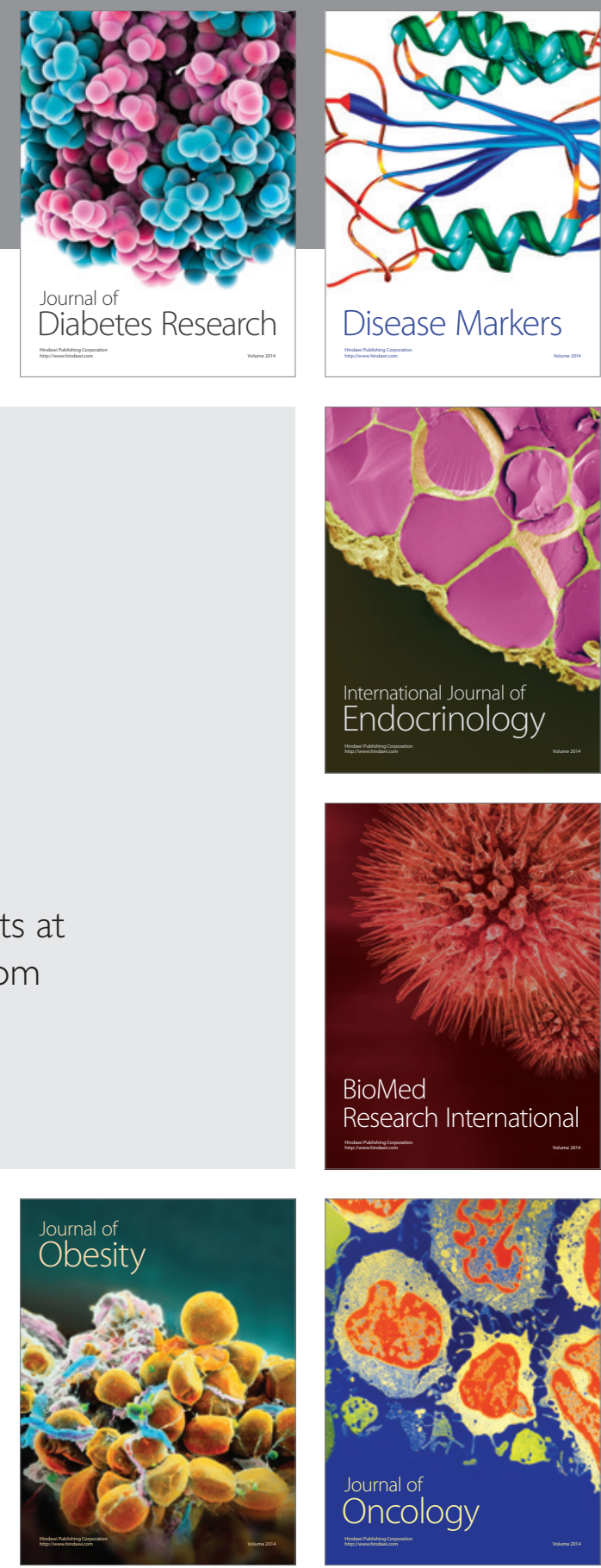

Disease Markers
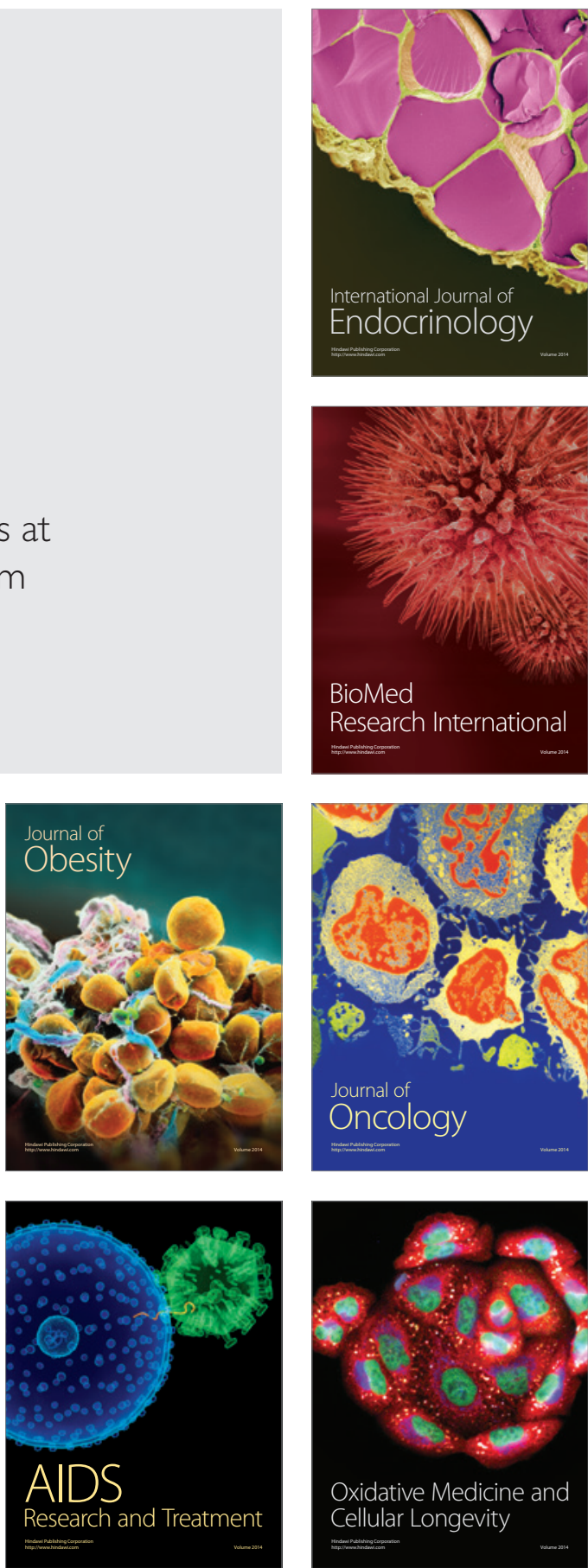